\title{
Pattern of intravenous immunoglobulins (IVIG) use in a pediatric intensive care facility in a resource limited setting
}

\author{
Nermeen M Galal \\ Medical Doctorate of Paediatrics, Cairo University, Cairo, Egypt
}

\begin{abstract}
Background: Intravenous Immunoglobulin (IVIG) preparations are scarce biological products used for replacement or immunomodulatory effects. Guidelines have been issued by regulatory health authorities to ensure provision of the products for patients who are in severe need.

Objectives: The study aimed at description of the pattern of IVIG use (label/off label indications), adverse effects observed, reason for choice of IVIG among other modalities and efficacy in a pediatric intensive care setting.

Methods: A retrospective chart review. Patients: The study included 45 cases admitted from 2008 through 2011 in a Pediatric Intensive Care Unit (PICU) of a tertiary referral pediatric hospital.

Results: The clinical diagnoses included neurology (35\%), neonatology (16\%), hematology (11\%), autoimmune disorders $(11 \%)$ immunodeficiency disorders (11\%), infections other than neonatal sepsis $(9 \%)$ and cardiology $(6.5 \%)$.

The indications for IVIG use had an Evidence category Ia / Ib in $62 \%$ of cases whereas the other $38 \%$ had level II and III evidence. Choice of IVIG as a therapeutic option was based on failure of other treatment options to achieve response in $46.5 \%$, lack of alternative treatment options $15.5 \%$ and the need for urgent response in $38 \%$. Adverse events, duration and doses are reported.

Conclusion: IVIG use is governed by availability of alternative options and the need for urgent response in critically ill children. Guidelines should be issued based on locally available treatment options and their cost effectiveness.

Keywords: Intravenous Immunoglobulin-Pediatrics- critically ill children

African Health Sciences 2013; 13(2): 261 - 265 http://dx.doi.org/10.4314/ahs.v13i2.9
\end{abstract}

\section{Introduction}

Intravenous Immunoglobulin (IVIG) preparations are scarce biological Products prepared by fractionation of pooled plasma with viral inactivation and screening for potential blood borne pathogens. IVIG products differ regarding their constituents (preservatives and inhibitors of $\operatorname{IgG}$ aggregation) and physical/chemical characteristics and adverse event causation (e.g. lyophilized powder or liquid and $\mathrm{pH})^{1}$. Attempts at rationalizing use because of scarcity and cost were issued by several regulatory authorities and expert panels like Federal Drug Association (FDA), American Academy of Allergy and Immunology, Comité d'évaluation et de diffusion des innovations technologiques (CEDIT) ${ }^{2,3,4}$

*Corresponding author:
Nermeen M.Galal
Medical Doctorate of Paediatrics, FRCPCH
Associate Professor of Paediatrics
Cairo University
1 A Road 233 Degla Maadi
Cairo, Egypt
Phone:+201001643197
Email: nermeengalal@gmail.com

African Health Sciences Vol 13 Issue 2 June 2013
Federal Drug Association approved IVIG indications include primary immunodeficiency disease, didiopathic/immune-mediated thrombocytopenic purpura (ITP), human immunodeficiency virus, bone marrow transplantation, Kawasaki disease and chronic lymphocytic leukemia ${ }^{4}$.

Off- label indications include several hematological, neurological, autoimmune and other disorders like multiple sclerosis, graft-versus-host disease in transplant patients, and prevention of antiphospholipid syndrome in miscarriage, severe asthma, Guillain-Barré syndrome, and progression of human immunodeficiency virus after delivery ${ }^{5}$.

Mechanism of action is either as a substitute of missing antibodies at replacement doses (200$400 \mathrm{mg} / \mathrm{kg} / \mathrm{month}$ ) in the treatment of primary and secondary antibody deficiencies. High-dose immunoglobulin (hdIVIG) given at doses of up to $2 \mathrm{~g} / \mathrm{kg} /$ day has immunomodulatory action mediated via a number of different effects like autoantibody neutralization and Interleukin 12 production ${ }^{6}$.

Reported side effects include: fever, headache, chills, myalgia, nausea and hypotension ${ }^{7}$. 
Substances such as prostaglandins, platelet-activating factor, and cytokines (e.g., tumor necrosis factoralpha, interleukin-6) have been described to cause reactions such as fever, bronchospasm, and changes in blood pressure, which are characteristic adverse events of IVIG treatment. Another possibility for the release of mediators is that in patients with infections, the antibodies infused react with circulating microbial antigens to form immune complexes, which again trigger the release of the respective mediators ${ }^{8}$. Other rare adverse events as thromboembolic or renal complications are associated with higher doses and fortunately are not common in children.

The study was aimed at determination of the pattern of IVIG use (label/off label indications), adverse effects observed, reason for choice of IVIG among other modalities if present and efficacy in a pediatric intensive care setting.

\section{Methods}

The study was conducted in a Pediatric Intensive Care Unit (PICU) of a tertiary referral pediatric hospital located in Cairo from 2008-2011 after Institutional review board's approval was obtained.

Study design: This was a retrospective chart review

Study population: Pediatric patients receiving IVIG for various indications during their intensive care unit stay.

Main study parameters: Demographic data, admission diagnosis, indication for PICU admission, indication of IVIG use, side effects, and doses of IVIG prescribed duration, outcome and availability of alternative therapies.

\section{Results}

The study included 45 cases admitted from 2008 through 2011. The age ranged from day $1-13$ years (Median 3.5 years). There were twenty four males and twenty one females.

The clinical diagnoses included neurology (35\%), neonatology (16\%), hematology (11\%), autoimmune disorders (11\%) immunodeficiency disorders (11\%), infections other than neonatal sepsis $(9 \%)$ and cardiology $(6.5 \%)$.

Admission diagnosis included twelve cases with Gillian Barre Syndrome (GBS), four cases with other neurological disorders (two with Acute Disseminated Encephalomyelitis (ADEM) and two with viral Encephalitis).Neonatal cases included seven ex preterm babies treated for neonatal sepsis. Hematological cases included five children with idiopathic thrombocytopenic purpura cases (ITP) .As for autoimmune disorders there were two cases of Kawasaki disease, three with Steven Johnson Syndrome (SJS following lamotrigine in two and sulphonamide in one child) Primary Immunodeficiency disorders (PID) included five primary immunodeficiency cases (one with Hyper IgE syndrome, two with Burtons' agammaglobulinemia and two with Common Variable Immunodeficiency. Infections other than neonatal sepsis included one patient with fulminant CMV infection (pneumonitis and hepatitis) and three with bacterial septic syndrome (Escherichia coli , Pseudomonas aeruginosa, and Staphylococcal infections). Cardiac cases included three children with acute viral myocarditis. Indications for NICU/PICU admission were progressive paralysis involving respiratory muscles and aspiration pneumonia in GBS patients and worsening Glasgow Coma Scale in the other neurological disorders. ITP cases (dropping counts with active bleeding, splenectomy, expanding intrabdominal hematoma with hypotension and CNS bleed), Autoimmune disorders .SJS (Extensive body involvement with surgical debridement of gangrenous patches, TPN hyper alimentation). for PID cases (three admissions with Pneumonia, one with severe immune enteritis and liver cell failure in HIES). The seven ex preterms were all admitted with sepsis (Group B Streptococcus, Escherichia coli,Staphylcoccus aurues , Streptococcus pneumoniae and Hemophilus Influenzae).

The indications for IVIG use had an Evidence category $\mathrm{Ia} / \mathrm{Ib}$ in $62 \%$ of cases whereas the other $38 \%$ had level II and III evidence. Details of indication for use / evidence category are demonstrated in Table 1. Categories of evidence are classified as :

Ia Evidence from meta-analysis of randomised controlled trails

Ia Evidence from at least one randomized controlled trial

IIa Evidence from at least one controlled study without randomization

IIb Evidence from at least one other type of quasiexperimental study

III Evidence from non-experimental descriptive studies, such as comparative studies, correlation studies and case control studies

IV Evidence from expert committees' reports or opinions and/or clinical experience of respected authorities. African Health Sciences Vol 13 Issue 2 June 2013 
Table 1: Use of Intravenous Immunoglobulin by diagnosis and evidence category

\begin{tabular}{lll}
\hline Diagnosis & $\begin{array}{l}\text { Number } \\
\text { of cases }\end{array}$ & $\begin{array}{l}\text { Evidence } \\
\text { category }\end{array}$ \\
\hline Guillian Barre syndrome & 12 & $\mathrm{Ia}$ \\
Acute dissemeniated Encephalomyelitis & 2 & $\mathrm{Ib}$ \\
Viral Encephalitis & 2 & $\mathrm{III}$ \\
Ex-preterm cases with neonatal sepsis & 7 & $\mathrm{Ia}$ \\
Idiopathic thrombocytopenic purpura & 5 & $\mathrm{Ia}$ \\
Kawasaki disease & 2 & $\mathrm{Ia}$ \\
Steven Johnson Syndrome & 3 & $\mathrm{II}$ \\
Agammaglobulinemia & 2 & $\mathrm{IIb}$ \\
Common variable Immunodeficiency & 2 & $\mathrm{IIb}$ \\
Hyper Immunoglobulin E Sydrome & 1 & $\mathrm{III}$ \\
Cytomegalovirus infection & 1 & $\mathrm{II}$ \\
Bacterial sepsis & 3 & $\mathrm{III}$ \\
Acute viral myocarditis & 3 & $\mathrm{III}$ \\
\hline
\end{tabular}

The choice of IVIG as a therapeutic option was based on failure of other treatment options to achieve response in $46.5 \%$, lack of alternative treatment options $15.5 \%$ and the need for urgent response in $38 \%$.

Adverse events recorded included fever in $29 \%$ $(\mathrm{n}=39)$, headache in $1.5 \% \quad(\mathrm{n}=2)$, chills in $2 \%$ $(\mathrm{n}=3)$, myalgia in $2 \%(\mathrm{n}=3)$, nausea in $3.6 \%(\mathrm{n}=5$ ) and hypotension in $0.7 \%(\mathrm{n}=1)$ through one hundred thirty six infusions.

Adverse events were managed by reducing infusion rate, administration of antihistaminic drugs /antipyretics and or stopping the infusion.

Two brands of IVIG were used alternatively based on availability in the hospital's pharmacy. No difference was noted statistically between the two preparations regarding adverse events 'causation.

The dose administered was $400 \mathrm{mg} / \mathrm{kg}$ in most cases except Kawasaki where a dose of $2 \mathrm{~g} / \mathrm{kg}$ was given. The administration period ranged between single administrations to 5 day courses.

Outcome measures: mortality was recorded in 13 $\%$ ( $\mathrm{n}=6$ - three cases diagnosed with septic syndrome and three cases with neonatal sepsis), complete resolution in $40 \%(\mathrm{n}=18)$, sequelae in the form of neurological disability (primary neurological disease), lung abnormalities (PID cases) and or chronic morbidities in $47 \%(\mathrm{n}=21)$.

\section{Discussion}

IVIG prescription should be done thoughtfully especially in resource limited settings with lack of guidelines of clear indications. Off label use of IVIG happens worldwide, an audit of IVIG use revealed a greater than expected proportion of non-category 1 IVIG use 9 . The ICU is a challenging setting as interventions may be life saving whereas the critically ill patients may be prone to develop complications. A study of IVIG use in an adult ICU revealed 19\% of IVIG prescriptions were for 'appropriate' indications and 7\% were 'inappropriate'. The remaining $74 \%$ were prescribed for indications with some evidence to support their use ${ }^{10}$.

In this study GBS was a common indication despite the presence of other therapeutic alternatives.A study reported that, in children with GBS requiring mechanical ventilation, Plasmapaharesis was superior to IVIG regarding the duration of Mechanical ventilation but not PICU stay or the short term neurological outcome ${ }^{11}$. As for cost In GBS patients, direct costs of IVIG therapy was found to be more than twice that of plasma exchange. Given equivalent efficacy and similar severity and frequencies of adverse events, plasmapaharesis if feasible is preferred ${ }^{12}$. However plasmapaharesis execution was often hindered by availability of filters and or small patient size.

In case of infections likely beneficial mechanisms of IVIG include improvement of serum bactericidal activity caused by neutralizing and opsonizing IgG and IgM antibodies, as well as stimulation of phagocytosis and neutralization of bacterial toxins 13.

The only FDA approved indications of IVIG use in infections so far are replacement for Preterms or Cytomegalovirus infection in transplanted cases. The cumulative evidence, along with the cost-effectiveness and risk of complications, 
must be considered when using IVIG to treat infection ${ }^{4}$.

The treatment effect of IVIG on mortality for patients with severe sepsis was borderline significant with a large degree of heterogeneity in treatment effect between individual studies as reported by Soares and colleagues ${ }^{14}$.Another Cochrane review revealed a significant mortality benefit when IVIG was compared with placebo in sepsis (RR 0.81; 95\% CI 0.70-0.93). However, analysis of trials with a low risk of bias yielded no reduction in mortality (RR 0.97 ; $95 \%$ CI $0.81-1.1^{15}$.

As for neonatal sepsis there was no difference regarding mortality, major or non major disability or of adverse events ${ }^{16}$ highlighting the advocated use as a replacement therapy before sepsis occurs. Should investment in IVIG as a treatment option for preterms with neonatal sepsis be decided, it is better started as a replacement to improve outcome and minimize complications.

Use of IVIG in ITP was definitely beneficial when compared to steroids but has definite indications, in patients who do not go into an immediate remission and where it is anticipated that remission may still occur and steroid-sparing agents are required ${ }^{17}$.

\section{Limitations of the study}

Comparing IVIG therapy to other treatment options within the same case as it was not feasible to make comparisons with other forms of treatment.

Overall the use of IVIG was supported by strong evidence in most situations given the existing circumstances. The most satisfactory results regarding achievement of therapeutic goal were obtained in cases of PID, ITP and GBS. The routine use in cases of established sepsis and neurological disorders other than GBS needs further supporting evidence especially with limited availability.

\section{Conclusion}

IVIG use should be rationalized to ensure availability for patients who need it the most especially in case of effective alternative treatment options. Guidelines for use should be issued based on locally available treatment options.

\section{References}

1. Feldmeyer L, Benden C, Haile SR, Boehler A, Speich R, French LE, Hofbauer GF. Not all intravenous immunoglobulin preparations are equally well tolerated. Acta Derm Venereol. 2010; 90(5):494-7.

2. Guillevin L.Indications for immunoglobulins. Recommendations of CEDIT of AP-HP in 2006.Rev Med Interne. 2007;28 Spec No. 1:1-3.

3. Stangel M, Gold R. Administration of intravenous immunoglobulins in neurology. An evidence-based consensus: update 2010. Nervenarzt. 2011 Apr;82(4):415-6..

4. Orange JS, Hossny EM, Weiler CR, Ballow M, Berger M, Bonilla FA, Buckley R, Chinen J, ElGamal Y, Mazer BD, Nelson RP Jr, Patel DD, Secord E, Sorensen RU, Wasserman RL, Cunningham-Rundles C; Primary Immunodeficiency Committee of the American Academy of Allergy, Asthma and Immunology. Use of intravenous immunoglobulin in human disease: a review of evidence by members of the Primary Immunodeficiency Committee of the American Academy of Allergy, Asthma and Immunology. J Allergy Clin Immunol. 2006;117(4 Suppl):S525-53.

5. Leong H, Stachnik J, Bonk ME, Matuszewski KA. Unlabeled uses of intravenous immune globulin. Am J Health Syst Pharm. 2008;65(19):1815-24.

6. El-Shanawany T, Jolles S. Intravenous immunoglobulin and autoimmune disease. Ann N Y Acad Sci. 2007; 10:507-15.

7. Brennan VM, Salomé-Bentley NJ, Chapel HM. Immunology Nurses Study. Prospective audit of adverse reactions occurring in 459 primary antibody-deficient patients receiving intravenous immunoglobulin. Clin Exp Immunol 2003; 133:247-51.)

8. Eibl MM .History of immunoglobulin replacement.. Immunol Allergy Clin North Am - 01NOV-2008; 28(4): 737-64,

9. Lin MW, Kirkpatrick PE, Riminton DS. How intravenous immunoglobulin is used in clinical practice: audits of two Sydney teaching hospitals. Intern Med J.2007;37(5):308-14.

10. Foster R, Suri A, Filate W, Hallett D, Meyer J, Ruijs T, Callum JL, Sutton D, Mehta S. Use of intravenous immune globulin in the ICU: a retrospective review of prescribing practices and patient outcomes. Transfus Med. 2010 Dec;20(6):403-8. doi: 10.1111/j.13653148.2010.01022.x.

11. El-Bayoumi MA, El-Refaey AM, Abdelkader AM, El-Assmy MM, Alwakeel AA, El-Tahan 
HM. Comparison of intravenous immunoglobulin and plasma exchange in treatment of mechanically ventilated children with Guillain Barré syndrome: a randomized study. Crit Care. 2011;15(4):R164.

12. Winters JL, Brown D, Hazard E, Chainani A, Andrzejewski C Jr. Cost-minimization analysis of the direct costs of TPE and IVIg in the treatment of Guillain-Barré syndrome. BMC Health Serv Res. 2011;11:101.

13. Christensen RD, Brown MS, Hall DC, Lassiter HA, Hill HR. Effect on neutrophil kinetics and serum opsonic capacity of intravenous administration of immune globulin to neonates with clinical signs of early-onset sepsis. J Pediatr 1991;118:606-14.

14. Soares M, Welton N, Harrison D, Peura P, Hari M, Harvey S, Madan J, Ades A, Palmer S, Rowan $\mathrm{K}$. An evaluation of the feasibility, cost and value of information of a multicentre randomised controlled trial of intravenous immunoglobulin for sepsis (severe sepsis and septic shock): incorporating a systematic review, meta-analysis and value of information analysis. Health Technol Assess. 2012;16(7):1-186.

15. Alejandria MM, Lansang MA, Dans LF, et al: Intravenous immunoglobulin for treating sepsis and septic shock. Cochrane Database Syst Rev 1. 2002;CD001090

16. Oba Y, Iwata K.Treatment of neonatal sepsis with intravenous immune globulin. N Engl J Med . 2011; 365(13): 1201-11.

17. Cooper N. Intravenous immunoglobulin and anti-RhD therapy in the management of immune thrombocytopenia. Hematol Oncol Clin North Am - 01-DEC-2009; 23(6): 1317-27. 